(2) Mobilisierung und Gewinnung von Unterstützung: Sie bietet infolgedessen auch bessere Möglichkeiten, die negative Betroffenheit und die Anliegen von spezifischen Gruppen zum Thema öffentlicher Erörterungen zu machen.

(3) Interessendurchsetzung durch Geben und Nehmen: Sie ermöglicht Verhandlungen und Tauschgeschäfte zugunsten partikularer, nicht-ökonomisierbarer Ansprüche.

Frank-Walter Steinmeier hat die Einrichtung deliberativer Gremien wie folgt begründet: „Konsenssuche wird hier zu einem dynamischen Prozess, in dessen Verlauf man traditionelle Blockaden überwindet, und dafür sorgt, dass sich in komplexen Entscheidungsprozessen die Waagschale im richtigen Moment zugunsten der Erneuerung senkt. "85 Es dürfte deutlich geworden sein, welche Anliegen und Interessen die Gewinner einer derart ausgestalteten Erneuerung wären. Wer den Grundsätzen der Gleichheit, Offenheit, Pluralität, ökonomischen Unabhängigkeit und prinzipiellen Inklusivität des Politischen anhängt, sollte sein Augenmerk deshalb auf Verbesserungsmöglichkeiten in der Ausgestaltung klassischer Repräsentationsformen legen. Die Fiktion von der umfassenden Einbindung qua rationaler Deliberation erweist sich nämlich als Trugschluss. Die praktische Umsetzung des Paradigmas ist geeignet, den eigenen Anspruch emanzipatorischer Demokratietheorie in sein Gegenteil zu verkehren.

\title{
Chancengleichheit oder Kooptation? Der Zugang kleiner Parteien zur Bundestagswahl
}

\author{
Florian Meinel
}

Das Recht der „kleinen Parteien“ oder „Splitterparteien“ führte - abgesehen von der Frage der Parteienfinanzierung - lange Zeit ein Schattendasein und dies, obwohl das Bundesverfassungsgericht sich zumindest verbal früh zur Bedeutung auch des außerparlamentarischen Parteienspektrums bekannt hat. ${ }^{1}$ Das konnte indes nur so bleiben, solange die Kleinen klein blieben, solange im Deutschen Bundestag regelmäßig nicht mehr als vier Parteien vertreten waren und solange dadurch eine relativ sichere Regierungsbildung gewährleistet war.

Die viel erörterte Krise der Volksparteien hat diese Situation grundlegend verändert. Nachdem sich durch die Konsolidierung der Linkspartei im Bundestag mittelfristig eine Fünfparteienkonstellation abzeichnet, werden Koalitions- und Regierungsbildung künftig schwieriger und unvorhersehbarer werden. Dass sich unter diesen Umständen der Kampf zwischen den etablierten und den kleinen Parteien verschärft, ist unvermeidlich. Die Versuchung wächst, neuen Parteien durch eine gezielte Gestaltung und Anwendung des Parteienrechts den Zugang zur Wahl zu versperren. Spätestens die durch nur fünftausend Zweitstim-

1 Vgl. BVerfGE 1, S. 208 (S. 255); BVerfGE 7, S. 99 (S. 107 f.). 
men zugunsten der SPD entschiedene Bundestagswahl 2002 dürfte allen Beteiligten die praktische Relevanz des Konflikts deutlich gemacht haben. Hätte damals nicht eine politische Eintagsfliege, die „Schill-Partei“, der Union ein knappes Prozent der Wählerstimmen abgerungen, hätten diese vielleicht als größte Fraktion den Anspruch auf die Kanzlerschaft stellen können. Ähnliche Konstellationen sind auch in Zukunft denkbar. So könnte, um nur zwei beliebige Beispiele zu nennen, schon ein kleiner Stimmenanteil der neu gegründeten Piratenpartei oder ein paar Dezimalstellen für eine Spaßpartei der FDP oder den Grünen und damit gleichzeitig ihren potentiellen Koalitionspartnern den Weg zur Macht verbauen.

Die Erfahrungen mit der staatlichen Parteienfinanzierung haben immer wieder gezeigt, dass die Benachteiligung der außerparlamentarischen Parteien zu den raren Zielen gehört, über die im Bundestag fraktionsübergreifend zumeist erstaunliche Einigkeit herrscht. ${ }^{2} \mathrm{Un}$ ter diesen Umständen ist es an der Zeit, auch das wahlrechtliche Problem der Zulassung von Kleinparteien zu Bundestags- und Landtagswahlen neu zu bedenken, das im Bundestagswahlkampf 2009 virulent geworden ist und über das das BVerfG vermutlich bald zu entscheiden haben wird.

\section{Das Wahlvorbereitungsverfahren und die „kleinen Parteien“}

Die Stellung einer politischen Partei im Wahlvorbereitungsverfahren unterscheidet sich zunächst vor allem danach, ob sie bereits parlamentarisch etabliert ist. ${ }^{3}$ War sie seit der letzten Wahl im Bundestag oder in einem Landtag mit wenigstens fünf Abgeordneten vertreten ( $\$ 18$ II BWahlG), so ist sie insofern privilegiert, als sie ohne weitere Prüfung bei den Landeswahlleitern Landeslisten einreichen kann (\$27 BWahlG). War sie bisher in keinem Parlament vertreten, muss sie zunächst beim Bundeswahlleiter fristgerecht eine Beteiligungsanzeige einreichen ( $\$ 18$ II 1 BWahlG). Diese Differenzierung zwischen etablierten und nicht etablierten Parteien ist zwar im Hinblick auf die formale Chancengleichheit der Parteien ${ }^{4}$ nicht unbedenklich, gehört aber zu den im Parteienstaat offenbar unvermeidlichen ${ }^{5}$ legalen Prämien auf den Machtbesitz, und man wird sie deshalb mit dieser oder jener Begründung hinzunehmen haben. ${ }^{6}$ Das BVerfG jedenfalls sieht sie durch einen „zwingenden Grund“, und zwar die Gefahr der Parteienzersplitterung, gerechtfertigt. ${ }^{7}$

2 Vgl. Michael Stolleis, Parteienstaatlichkeit - Krisensymptome des demokratischen Verfassungsstaates?, in: VVDStRL 44 (1986), S. 7 (S. 30 ff.).

3 Hierzu und zum Folgenden auch Eike Michael Frenzel, Das Erfordernis der Anerkennung als Partei nach $₫ 18$ BWG. Zugleich Anmerkung zu den Beschlüssen des BVerfG im Vorfeld der Bundestagswahl 2009, in: NVwZ 2009, S. 1349 ff.

4 Vgl. BVerfGE 14, S. 121 (S. 132 ff.); BVerfGE 24, S. 300 (S. 340 f.); BVerfGE 44, S. 125 (S. 146); BVerfGE 85, S. 264 (S. 297 f.); BVerfG, in: NVwZ 2008, S. 991 (S. 993); zuletzt BVerfG, Urteil vom 21. April 2009, 2 BvC 2/06, Rn. 84; Dieter Grimm, Politische Parteien, in: Ernst Benda / Werner Maihofer / Hans-Jochen Vogel (Hrsg.), Handbuch des Verfassungsrechts der Bundesrepublik Deutschland, Berlin 1994, \$14, Rn. $42 \mathrm{ff}$.

5 Siehe die Länderberichte in: Rüdiger Wolfrum / Gunnar Schuster (Hrsg.), Verfahren der Kandidatenaufstellung und der Wahlprüfung im europäischen Vergleich, Baden-Baden 1994.

6 Vgl. Wolfgang Schreiber, Handbuch des Wahlrechts zum Deutschen Bundestag, Köln 2002, \$18 BWG, Rn. 9; ders., Wahlkampf, Wahlrecht und Wahlverfahren, in: Hans-Peter Schneider I Wolfgang Zeh (Hrsg.), Parlamentsrecht und Parlamentspraxis, Berlin 1989, \$12, Rn. 46 ff.

7 Vgl. BVerfG, in: NVwZ 1994, S. 157. 
Die eigentliche Problematik des Verfahrens liegt nicht in dem formalen Erfordernis der Beteiligungsanzeige, sondern in der ihr nachgelagerten Sachentscheidung. Nach der Beteiligungsanzeige nämlich hat der Bundeswahlausschuss zu prüfen, ob die anzeigende Partei überhaupt als Partei anzuerkennen ist (\$ $18 \mathrm{IV}$ Nr. 2 BWahlG). Für die Anerkennung reicht dabei die einfache, für die Ablehnung ist eine Zweidrittelmehrheit erforderlich. Die Entscheidung ergeht spätestens 72 Tage vor der Wahl (\$18 IV BWahlG). Prüfungsmaßstab der Entscheidung des Bundeswahlausschusses ist der Parteienbegriff aus $₫ 2$ PartG, wonach eine Partei eine Vereinigung von Bürgern ist, die dauernd oder für längere Zeit für den Bereich des Bundes oder eines Landes auf die politische Willensbildung Einfluss nehmen und an der Vertretung des Volkes im Deutschen Bundestag oder einem Landtag mitwirken will, wenn sie nach dem Gesamtbild der tatsächlichen Verhältnisse, insbesondere nach Umfang und Festigkeit ihrer Organisation, nach der Zahl ihrer Mitglieder und nach ihrem Hervortreten in der Öffentlichkeit eine ausreichende Gewähr für die Ernsthaftigkeit dieser Zielsetzung bietet. ${ }^{8} \mathrm{Ob}$ demnach die beteiligungswillige Gruppierung eine Partei ist, entscheidet der Bundeswahlausschuss in freier Beweiswürdigung. ${ }^{9}$ Die Parteieigenschaft wird also nicht etwa widerleglich vermutet. Den Parteien werden in der Praxis vom Bundeswahlleiter vielmehr nicht unerhebliche Mitwirkungs- und Dokumentationspflichten auferlegt.

Kommt der Bundeswahlausschuss hinsichtlich der Parteieigenschaft zu einem positiven Ergebnis, entscheiden die Landeswahlausschüsse spätestens 58 Tage vor der Wahl über die Zulassung der von der Partei eingereichten Landeslisten. Deren Einreichung ist für parlamentarisch nicht etablierte Parteien ebenfalls an strenge Voraussetzungen gebunden. Anders als die etablierten Parteien können sie Landeslisten nur einreichen, wenn diese in der Regel von mindestens 2.000 Wahlberechtigten des betreffenden Bundeslands unterschrieben worden sind ( $\$ 27$ I BWahlG). Diese Quorenregelung soll dazu dienen, nicht ernstgemeinte und offensichtlich aussichtslose Bewerbungen zu verhindern und ist trotz zum Teil sehr heftiger Kritik $^{10}$ vom BVerfG immer wieder bestätigt worden. ${ }^{11}$ Sind auch die übrigen förmlichen Voraussetzungen erfüllt ( $\$ 27$ II - V BWahlG), lässt der Landeswahlausschuss die Landesliste zu. Wenn er ihr wegen formeller Mängel die Zulassung versagt, kann diese Entscheidung vor dem Bundeswahlausschuss angefochten werden ( $\$ 28$ II 1 BWahlG).

Stellt dieser die Parteieigenschaft dagegen mit der erforderlichen Zweidrittelmehrheit nicht fest, ist das Verfahren für die beteiligungswillige Partei sofort beendet. Sie bleibt von der Bundestagswahl ausgeschlossen, denn die Feststellung bindet nach $\$ 18$ IV BWahlG alle Wahlorgane, also auch die Landeswahlausschüsse. Da Landeslisten jedoch nur von Parteien eingereicht werden können ( $\$ 27$ I 1 BWahlG), ist jede trotz der Entscheidung des Bundeswahlausschusses eingereichte Landesliste in subjektiver Hinsicht ipso jure formell fehlerhaft. Die negative Entscheidung über die Parteieigenschaft führt also notwendig zur Ablehnung aller Landeslisten der betroffenen Partei. Auch versagt in dieser Konstellation

8 Ausführlich dazu Martin Morlok, in: Horst Dreier (Hrsg.), Grundgesetz, Bd. II, Tübingen 2006, Art. 21, Rn. 28 ff.; Philip Kunig, in: Wolfgang Isensee / Paul Kirchhof (Hrsg.), Handbuch des Staatsrechts, Bd. III, Heidelberg 2005, $\$ 40$, Rn. 6 ff.

9 Wolfgang Schreiber, a.a.O. (Fn. 6), \$18 BWG, Rn. 18.

10 Siehe nur Hans Meyer, in: Wolfgang Isensee / Paul Kirchhof (Hrsg.), a.a.O. (Fn. 8), \$46, Rn. 58 ff.; Jochen A. Frowein, Die Rechtsprechung des Bundesverfassungsgerichts zum Wahlrecht, in: AöR 99 (1974), S. 72 (S. 97).

11 BVerfGE 3, S. 19 (S. 29 ff.); BVerfGE 12, S. 10 (S. 27); BVerfGE 24, S. 300 (S. 341); BVerfGE 41, S. 399 (S. 421); BVerfGE 71, S. 81 (S. 96 f.). 
die nach $₫ 18$ II 1 BWahlG an sich statthafte Beschwerde zum Bundeswahlausschuss. Hier beißt sich die Katze in den Schwanz, müsste doch der Bundeswahlausschuss, um der Beschwerde abzuhelfen, seine eigene Entscheidung über die Parteieigenschaft korrigieren, um so die Bindungswirkung zu beseitigen. Dazu ist er jedoch aus mehreren Gründen nicht berechtigt: Zum einen sieht das Gesetz ein Widerspruchsverfahren insoweit nicht vor. Zum anderen könnte er gar nicht erneut entscheiden, ohne dadurch die 72-Tage-Frist des $₫ 18$ IV BWahlG zu verletzen.

\section{Zur Rechtsstellung des Bundeswahlausschusses}

Nach alledem ist deutlich, über welche Machtfülle der Bundeswahlausschuss bei der Zulassung kleiner Parteien verfügt. Diese Bedeutung ergibt sich nicht nur aus der formellen und materiellen Bindungswirkung seiner Feststellungsentscheidung. Sie findet in der neueren Staatspraxis ihre Bestätigung. So erkannte der Bundeswahlausschuss jüngst von 49 Gruppierungen, die eine Beteiligungsanzeige für die am 27. September 2009 stattfindende Bundestagswahl abgegeben hatten, nur 21 als Parteien an, versagte einer deutlichen Mehrheit von 28 dagegen - jeweils einstimmig - die Anerkennung. ${ }^{12}$ Davon betroffen waren durchaus nicht nur Neulinge wie beispielsweise die erst 2007 gegründete „Soziale Alternative für Gerechtigkeit" (SAG), sondern auch politische Vereinigungen, die in der Vergangenheit bereits an Wahlen teilgenommen hatten, so etwa die aus der Partei Graue Panther hervorgegangene Vereinigung „Die Grauen“, die „Anarchistische Pogo-Partei Deutschlands“ sowie die „Partei für Arbeit, Rechtsstaat, Tierschutz, Elitenförderung und basisdemokratische Initiative (Die PARTEI)“. Sie alle hatten sich bereits an der letzten Bundestagswahl beteiligt und waren von den Landeswahlleitern zu einigen Landtagswahlen zugelassen worden. Nachdem im Vorfeld der Bundestagswahl im Jahr 2005 jedoch teilweise heftige Kritik am damaligen Bundeswahlleiter geübt worden war, der die genannten Parteien anerkannt und sie damit in den Genuss anteiliger Sendeplätze im öffentlichen Rundfunk gebracht hatte ${ }^{13}$, scheinen die Prüfungskriterien unter seinem Amtsnachfolger strenger gehandhabt zu werden.

\subsection{Zur fehlenden Unabhängigkeit des Bundeswahlausschusses}

In auffälligem Widerspruch zu dieser umfassenden Entscheidungskompetenz des Bundeswahlausschusses steht seine institutionelle Struktur. Er besteht aus dem Bundeswahlleiter, dessen Amt nach gängiger Staatspraxis vom Präsidenten des Statistischen Bundesamtes bekleidet wird, und acht von ihm benannten Beisitzern (\$9 II BWahlG). Der Bundeswahlleiter wird nach $\$ 9$ I BWahlG vom Bundesminister des Innern ernannt, und zwar auf unbe-

12 Eine Aufzählung der Parteien, die ihre Teilnahme an der Bundestagswahl dem Bundeswahlleiter angezeigt haben, findet sich in der Pressemitteilung des Bundeswahlleiters vom 30. Juli 2009. Von den 49 Gruppierungen wurden 21 als Parteien anerkannt, 28 wurde die Anerkennung versagt (Pressemitteilung vom 17. Juli 2009). Beide Pressemitteilungen sind abrufbar unter www. bundeswahlleiter.de.

13 Vgl. Thierse und Schily üben heftige Kritik an TV-Werbespot der APPD, in: Das Parlament vom 12. September 2005. 
stimmte Zeit ( $\$ 1$ I BWahlO). Er kann deshalb auch jederzeit abberufen und ausgetauscht werden. ${ }^{14}$ Über seine Beisitzer ist im Bundeswahlgesetz nichts bestimmt, jedoch regelt $\$ 4$ II BWahlO, dass bei der Auswahl „in der Regel die Parteien in der Reihenfolge der bei der letzten Bundestagswahl [...] errungenen Zahlen der Zweitstimmen angemessen berücksichtigt und die von ihnen rechtzeitig vorgeschlagenen Wahlberechtigten berufen werden".

Die Organisation des Ausschusses muss aus mindestens zwei Gründen gravierenden verfassungsrechtlichen Bedenken begegnen: Erstens handelt es sich um ein der Kontrolle der amtierenden Bundesregierung, speziell des Bundesinnenministers unterstelltes Gremium. Wenn der Bundeswahlleiter jederzeit ausgetauscht werden kann und damit auch die Beisitzer neu benannt werden können, sind jederzeit politisch motivierte Interventionen in die Entscheidungsfindung möglich. Noch gewichtiger sind jedoch, zweitens, die Einwände gegen den Parteienproporz, den $\$ 4$ II BWahlO vorsieht. Ist nämlich der Bundeswahlausschuss ein von den Parteien - und zwar von den jeweils erfolgreichsten acht - dominiertes, mit Mehrheit entscheidendes Gremium, so ist das Feststellungsverfahren nach $\$ 18$ II BWahlG seinem Wesen nach nicht neutral, sondern es handelt sich um nichts anderes als ein Kooptationsverfahren. Mit anderen Worten: Wer in Deutschland bei Wahlen als Partei antreten darf, entscheidet im Streitfall die unmittelbare Konkurrenz. Wie es unter diesen Umständen um die Chancengleichheit tatsächlich bestellt ist, liegt auf der Hand.

\subsection{Zum Maßstab der Entscheidung}

Als problematisch erweist sich insoweit auch die denkbar unbestimmte Maßstabsnorm, anhand derer die Entscheidung zu treffen ist. Sie gibt dem Wahlausschuss die Frage an die Hand, ob die fragliche Vereinigung nach dem „Gesamtbild der tatsächlichen Verhältnisse“ eine ausreichende Gewähr für die Ernsthaftigkeit ihrer Zielsetzung bietet ( $\$ 2$ I PartG). Zum Gesamtbild gehören insbesondere der Umfang und die Festigkeit ihrer Organisation, die Zahl ihrer Mitglieder und ihr Hervortreten in der Öffentlichkeit. Diese gesetzlichen Kriterien sind jedoch nicht abschließend. ${ }^{15}$

Dem so definierten Begriff der politischen Partei wohnen in hohem Maße offene Elemente inne. ${ }^{16}$ „Umfang und Festigkeit der Organisation“ und das „Hervortreten in der Öffentlichkeit" sind vergleichsweise unbestimmte, der Wertung zugängliche Begriffe. Ihre Auslegung und zumal die Gewichtung der verschiedenen Kriterien zueinander eröffnen dem Ausschuss einen sehr weiten Entscheidungsspielraum. Wie für unbestimmte Rechtsbegriffe typisch, ist innerhalb eines gewissen Spektrums mit entsprechender Begründung vieles vertretbar. Selbst scheinbar harte Kriterien wie die Mitgliederzahl lassen sich nicht in dem Sinne festlegen, dass eine bestimmte Größe per se Gewähr für die Ernsthaftigkeit böte. ${ }^{17}$ Hinzu tritt die Gefahr, dass der Parteibegriff bei seiner Anwendung induktiv mit dem üblichen Erscheinungsbild der traditionellen großen Parteien mit Massenbasis angereichert wird.

14 Vgl. Wolfgang Schreiber, a.a.O. (Fn. 6), \$9 BWG, Rn. 4.

15 Vgl. BVerwG, in: NJW 1993, S. 3213 (S. 3214).

16 Vgl. Philip Kunig, a.a.O. (Fn. 8), $\$ 40$, Rn. 9 f.

17 Vgl. Philip Kunig, in: ders. / Ingo von Münch (Hrsg.), Grundgesetz-Kommentar, Bd. II, München 2001, Art. 21, Rn. 25. 
So konnte der Bundeswahlausschuss zuletzt etwa die Satirepartei „Die PARTEI“ als Nichtpartei einstufen ${ }^{18}$, obwohl sie über ein Vielfaches der Mitglieder verfügt, die andere gleichzeitig als Partei anerkannte Gruppierungen wie beispielweise die Zentrumspartei vorweisen können. ${ }^{19}$ Auch wurde ihr vom Ausschuss ein Mangel an Öffentlichkeitsarbeit entgegengehalten, obwohl sie als Gründung der Redaktion des Magazins „Titanic“ über ein monatliches Parteiorgan mit einer sechsstelligen Verkaufsauflage verfügt, was die Öffentlichkeitswirksamkeit aller anderen zugelassenen Kleinparteien bei weitem übertreffen dürfte. Gewiss entspricht diese Art des öffentlichen Auftretens nicht dem der größeren Parteien; doch darauf kann es im Rahmen von $\$ 2$ PartG nach allgemeiner Ansicht nicht ankommen. ${ }^{20}$ Der Verdacht, dass, gestützt auf die objektiven gesetzlichen Kriterien, unter der Hand doch die durch Art. 21 GG verbotene ${ }^{21}$ inhaltliche Kontrolle der von der Partei verfolgten Ziele stattfindet, kann auf diese Weise im Einzelfall zumindest naheliegen. Die OSZE hat die genannten Entscheidungen des Bundeswahlausschusses jedenfalls zum Gegenstand des Berichts über ihre Wahlbeobachtung in der Bundesrepublik gemacht. ${ }^{22}$

\subsection{Möglichkeiten einer verfassungskonformen Ausgestaltung des Verfahrens vor dem Bundeswahlausschuss}

Das deutsche Wahlrecht gibt mit der Fünf-Prozent-Hürde nach $\$ 6$ BWahlG den etablierten Parlamentsparteien das im Verhältniswahlsystem denkbar schärfste Schwert gegen Splitterparteien in die Hand. Gleichwohl durchzieht das Wahlrecht, wie gezeigt, auch unterhalb der Schwelle dieser Regelung eine gegen kleine Parteien gerichtete Tendenz, die neben $\$ 6$ BWahlG vielfach als grundlose Privilegierung der etablierten Parteien erscheinen muss. Inwiefern solche Sicherungen gegen nicht hinreichend ernsthafte Mitbewerber tatsächlich angezeigt sind, ist eine Frage des politischen Ermessens, für die sich der Verfassung keine substantiellen Aussagen entnehmen lassen. Soweit der Gesetzgeber allerdings an seiner derzeitigen Beurteilung des Gefährdungspotentials der „Parteienzersplitterung“ festzuhalten gedenkt, wird er zumindest das Verfahren vor dem Bundeswahlausschuss verfassungsgemäß auszugestalten haben.

Dazu gehörte insbesondere eine Neuregelung seiner Entscheidungsbefugnisse. Denn je niedriger die demokratische Legitimation eines Hoheitsträgers ist, desto höher muss die Steuerungstiefe des Gesetzes sein, das seine Befugnisse regelt. ${ }^{23}$ Danach kommt nur eine drastische Verringerung der Kompetenzen des Bundeswahlausschusses in Frage, denn erhöhen lässt sich die Legitimation des Ausschusses wohl kaum, da doch selbst ein direkt vom Bundestag bestelltes Gremium notwendigerweise vor allem die Interessen der im Parlament vertretenen Parteien wahrnehmen würde.

18 Vgl. Patrick Bahners, Ernst ist das Wählen - Lücke des Rechtsschutzes: Ist Die PARTEI eine Partei?, in: FAZ vom 31. Juli 2009, S. 33.

19 Die Sitzung des Bundeswahlausschusses ist bislang noch nicht amtlich protokolliert. Sie ist aber über das Parlamentsfernsehen des Deutschen Bundestages abrufbar (www.webtv.bundestag.de).

20 Vgl. statt aller Hans Hugo Klein, in: Theodor Maunz / Günter Dürig (Hrsg.), Grundgesetz. Kommentar, Art. 21, Loseblattsammlung, München 2009, Rn. 227.

21 Vgl. ebenda, Rn. 231.

22 Vgl. den Beitrag von Hans-Jörg Schmedes in diesem Heft der ZParl.

23 Vgl. Gunnar Folke Schuppert, Verwaltungswissenschaft, Baden-Baden 2000, S. 475 ff. 
Verfassungsmäßig könnte das Verfahren dadurch ausgestaltet werden, dass die gesetzlichen Vorgaben viel stärker als bisher formalisiert und auf diese Weise die wertenden Elemente der Entscheidung durch eine gesetzliche Feinsteuerung erheblich reduziert würden. Eher als die gegenwärtige wäre deshalb selbst eine Regelung zu rechtfertigen, die für eine beteiligungswillige Partei eine bestimmte Zahl von Mitgliedern verlangt. Vorzuziehen wäre indessen ein anderer Weg: Es ist dem Gesetzgeber in den Grenzen der Willkür unbenommen, das Quorum der erforderlichen Unterstützungsunterschriften nach $\$ 27$ I BWahlG stark anzuheben, zumindest wenn er im Gegenzug die materiellen Voraussetzungen weniger streng gestaltet. Dadurch hätten die Parteien es selbst in der Hand, den Nachweis ihrer Ernsthaftigkeit und ihres Rückhalts in der Bevölkerung zu führen.

\section{Das Rechtsschutzdefizit und seine Konsequenzen}

Eine solche weitgehende Formalisierung des Zulassungsverfahrens erscheint zumindest solange verfassungsrechtlich dringend geboten, wie die prozessuale Situation der abgelehnten Bewerber derart ungünstig ist. Eine negative Feststellungsentscheidung des Bundeswahlausschusses über die Parteieigenschaft ist zumindest vor der Wahl gerichtlich überhaupt nicht überprüfbar, eine Wahlprüfungsbeschwerde nach der Wahl ist in der Regel völlig zwecklos.

Über alle mit der Vorbereitung und Durchführung von Wahlen zusammenhängenden Fragen besitzt der Bundestag nach Art. 41 GG ein Höchstmaß an Autonomie; nicht nur gegenüber der Einflussnahme durch die Regierung, sondern auch gegenüber einer Kontrolle durch die Rechtsprechung. Das gesamte Wahlvorbereitungsverfahren und damit auch die Entscheidung des Bundeswahlausschusses nach $\$ 18$ BWahlG gehört zu den Gegenständen, die ausschließlich im Verfahren der Wahlprüfung nach Art. $41 \mathrm{GG}, \$ \$ 1 \mathrm{ff}$. WahlPrüfG und $\$ 48$ BVerfGG angefochten werden können. ${ }^{24}$ Das hat das BVerfG seit je so gesehen $^{25}$ und in zwei Kammerentscheidungen kürzlich noch einmal bekräftigt. ${ }^{26}$ Es behandelt Art. 41 II GG nicht nur als lex specialis gegenüber Art. 19 IV GG ${ }^{27}$, sondern auch als vorrangig gegenüber allen anderen verfassungsprozessualen Verfahrensarten. Demnach scheidet sowohl eine auf die Verletzung der Gleichheit und Öffentlichkeit der Wahl ge-

24 Vgl. Hans Hugo Klein, a.a.O. (Fn. 20), Art. 41, Rn. 60; Siegfried Magiera, in: Michael Sachs (Hrsg.), Grundgesetz. Kommentar, München 2009, Art. 41, Rn. 3; Hermann Rechenberg, in: Rudolf Dolzer / Klaus Vogel / Karin Graßhof (Hrsg.), Bonner Kommentar zum Grundgesetz, Heidelberg 1978, Art. 41, Rn. 6, 22; Heinrich Lang, in: Karl Heinrich Friauf / Wolfram Höfling (Hrsg.), Berliner Kommentar zum Grundgesetz, Berlin 2005, Art. 41, Rn. 25.

25 Vgl. BVerfGE 11, S. 329 (S. 329 f.); BVerfGE 14, S. 154; BVerfGE 16, S. 128 (S. 130); BVerfGE 22, S. 277; BVerfGE 28, S. 214; BVerfGE 29, S. 18; BVerfGE 63, S. 73; BVerfG, in: BayVBl 1999, S. 46.

26 Vgl. BVerfG, Beschluss vom 31. Juli 2009, 2 BvQ 45/09 (betraf die Partei „Die Grauen“); Beschlüsse vom 24. August 2009, 2 BvR 1898/09 („Freie Union“) und 2 BvQ 50/09 („Die PARTEI").

27 Vgl. BVerfGE 22, S. 277 (S. 281); BVerfGE 28, S. 214; BVerfGE 34, S. 81 (S. 94); BVerfGE 46, S. 196 (S. 198); BVerfGE 66, S. 232 (S. 234); Siegfried Magiera, a.a.O. (Fn. 24), Art. 41, Rn. 4; Wolfgang Löwer, in: Wolfang Isensee / Paul Kirchhof, a.a.O. (Fn. 8), \$70, Rn. 160; vgl. demgegenüber die im Vordringen befindliche andere Auffassung von Hans Meyer, a.a.O. (Fn. 10), $\$ 46$, Rn. 100; Wolf Rüdiger Schenke, Der gerichtliche Rechtsschutz im Wahlrecht, in: NJW 1981, S. 2440 ff.; Martin Morlok, a.a.O. (Fn. 8), Art. 41, Rn. 8, 12. 
stützte Verfassungsbeschwerde von einzelnen Wahlberechtigten ${ }^{28}$ als auch ein auf die Verletzung von Art. 21 GG gestütztes Organstreitverfahren der Partei als solcher aus; letzteres auch deshalb, weil der Bundeswahlausschuss kein tauglicher Antragsgegner im Sinne von Art. 93 I Nr. 1 GG ist. ${ }^{29}$ In Übereinstimmung mit dieser Auslegung von Art. 41 GG steht die Regelung des $\$ 49$ BWahlG, der alle anderen als die wahlprüfungsrechtlichen Rechtsbehelfe in seinem Anwendungsbereich ausschließt. Das BVerfG entnimmt Art. 41 GG zudem eine Verfassungsentscheidung für einen auf die Zeit nach der Wahl beschränkten Rechtsschutz in Wahlsachen. ${ }^{30}$ Danach soll auch ein im Hinblick auf eine spätere Wahlprüfungsbeschwerde gestellter Antrag nach $\$ 32$ BVerfGG prinzipiell ausgeschlossen sein. ${ }^{31}$ Dies gebiete der „reibungslose Ablauf einer Parlamentswahl“32.

Nach alledem kann eine fehlerhafte Entscheidung des Bundeswahlausschusses nur festgestellt werden, wenn gegen die Gültigkeit der Wahl von einem Wahlberechtigten Einspruch nach $₫ 2$ WahlPrüfG beim Bundestag eingelegt wird. ${ }^{33}$ Über diesen Einspruch entscheidet zunächst der Wahlprüfungsausschuss des Bundestages gemäß $₫ 3$ I WahlPrüfG. Gegen diese Entscheidung kann nach Art. 41 II GG und $\$ 48$ BVerfGG das BVerfG angerufen werden. Sowohl die parlamentarische Wahlprüfung als auch die Wahlprüfungsbeschwerde vor dem BVerfG dienen jedoch nach gefestigter Rechtsprechung ${ }^{34}$ und nahezu allgemeiner Ansicht in der Literatur ${ }^{35}$ nicht dem Schutz subjektiver Rechte aus Art. 38 und 21 GG, sondern ausschließlich der objektiven Richtigkeit der Zusammensetzung des Parlaments und damit der Durchsetzung des demokratischen Willens und der objektiven Wahlrechtsgrundsätze. Folgerichtig kann das gesamte Wahlprüfungsverfahren auch nur dann Erfolg haben, wenn festgestellte formelle oder materielle Fehler tatsächlich Einfluss auf die Zusammensetzung des Bundestages gehabt haben können, nicht dagegen schon bei der Verletzung von Wahlrechtsgrundsätzen im Einzelfall. ${ }^{36}$ Es will zwar nicht recht einleuch-

28 Vgl. BVerfGE 16, S. 128; BVerfGE 29, S. 18 (S. 19); BVerfGE 74, S. 96 (S. 101).

29 Vgl. BVerfGE 83, S. 156 (S. 157).

30 Vgl. BVerfGE 14, S. 154.

31 Vgl. BVerfG, Beschluss vom 28. November 1990, 2 BvQ 18/90; BVerfG, Beschluss vom 31. Juli 2009, 2 BvQ 45/09; andere Auffassung Hans Lechner / Rüdiger Zuck, Bundesverfassungsgerichtsgesetz, München 2006, $\$ 48$, Rn. 15; Bruno Schmidt-Bleibtreu, in: ders. I Theodor Maunz / Franz Klein / Herbert Bethge (Hrsg.), Bundesverfassungsgerichtsgesetz. Kommentar, $\$ 48$, München 2009, Rn. 38.

32 BVerfGE 16, S. 128 (S. 129); siehe auch HessVGH, in: DÖV 1966, S. 505.

33 Hierzu und zum Folgenden Andreas Ortmann, Probleme der Wahlprüfungsbeschwerde nach $\$ 48$ BVerfGG, in: ThürVBl 2006, S. $169 \mathrm{ff}$.

34 Vgl. BVerfGE 22, S. 277 (S. 281); BVerfGE 34, S. 81 (S. 94 f., S. 97); BVerfGE 34, S. 201 (S. 203); BVerfGE 48, S. 271 (S. 280); BVerfGE 59, S. 119 (S. 123); BVerfGE 66, S. 369; BVerfGE 79, S. 47 (S. 48); BVerfGE 85, S. 148.

35 Vgl. Peter Badura, Staatsrecht, München 2003, Rn. E 26; Hartmut Maurer, Staatsrecht, München 2005, \$ 13, Rn. 44; Hans Hugo Klein, a.a.O. (Fn. 20), Art. 41, Rn. 47; Klaus Schlaich / Stefan Korioth, Das Bundesverfassungsgericht, München 2007, Rn. 344; Ludger-Anselm Versteyl, in: Ingo von Münch / Philip Kunig, a.a.O. (Fn. 17), Art. 41, Rn. 8; Gerald Kretschmer, in: Hans-Peter Schneider / Wolfgang Zeh (Hrsg.), a.a.O. (Fn. 6), \$13, Rn. 1 ff.

36 Vgl. Bruno Schmidt-Bleibtreu, a.a.O. (Fn. 31), \$ 48 (2006), Rn. 39a; Hans Hugo Klein, a.a.O. (Fn. 20), Art. 41, Rn. 105 ff.; ausführlich David N. Rauber, Wahlprüfung in Deutschland, Baden-Baden 2005; Thorsten Koch, „Bestandsschutz“ für Parlamente? - Überlegungen zur Wahlfehlerfolgenlehre, in: DVBl 2000, S. 1093 ff.; für eine umfassende Subjektivierung des Wahlprüfungsrechts dagegen Heinrich Lang, Subjektiver Rechtsschutz im Wahlprüfungsverfahren, Berlin 
ten, dass der Zweite Senat des BVerfG einerseits Art. 38 I GG ein allgemeines subjektives öffentliches Recht auf Demokratie entnimmt ${ }^{37}$, andererseits aber konkrete Verletzungen dieses Rechts bei der Wahl weiterhin für nicht wehrfähig erachtet. Auch ist die schleppende Bearbeitung der Einsprüche durch den Bundestag ein verfassungsrechtlicher Missstand ersten Ranges. ${ }^{38}$ Doch de constitutione lata ist daran nichts zu ändern ${ }^{39}$, Art. 41 GG ist insoweit eindeutig. Folgerichtig haben Bundestag und BVerfG keinem der zu Tausenden an sie herangetragenen Einsprüche je zum Erfolg verholfen. ${ }^{40}$ Für „Splitterparteien“ ist die Wahlprüfung damit jedenfalls in der Regel völlig aussichtslos. ${ }^{41}$

Die grundgesetzlich festgeschriebene, weitreichende parlamentarische Autonomie folgt einer langen verfassungsgeschichtlichen Tradition ${ }^{42}$ und findet sich auch in den meisten deutschen Landesverfassungen. ${ }^{43}$ Sie fand im 19. Jahrhundert ihre Rechtfertigung in einem konstitutionellen Verständnis der Gewaltenteilung. Keinem exekutiven oder judikativen Organ sollte das Recht zukommen, über die Zusammensetzung der gesetzgebenden Körperschaft zu entscheiden. ${ }^{44}$ Völlig überzeugen konnte diese Begründung nur, solange ausschließlich das Parlament, nicht aber die anderen Staatsorgane die Idee der Volkssouveränität verkörperten. In der Parteiendemokratie, wie sie sich nach dem Ende der Monarchie entwickelt hat, ist die Problematik der Gewaltenteilung eine andere geworden. ${ }^{45}$ Nicht nur gegen den administrativen oder gerichtlichen Eingriff ist die Legitimation des Parlaments zu sichern, sondern auch gegen die Mediatisierung des Parlaments durch die Parlamentsparteien.

Trägt man dieser Entwicklung nicht genügend Rechnung, droht die Wahlprüfungshoheit des Parlaments ihren normativen Sinn völlig umzukehren: Von einer Sicherung der Gesetzgebung gegen die Exekutive wird sie zu einem Selbstbehauptungsmittel der den Bundestag dominierenden Parteien, zum Kooptationsrecht. Bereits die Weimarer Reichsverfassung löste sich deshalb partiell von der konventionellen Wahlprüfung durch den

1997; ders., in: Karl Heinrich Friauf/ Wolfram Höfling (Hrsg.), a.a.O. (Fn. 24), Art. 41 (2005), Rn. 58 ff.; kritisch zur herrschenden Meinung auch Hans-Peter Schneider, in: ders. / Erhard Denninger / Wolfgang Hoffmann-Riem / Ekkehart Stein (Hrsg.), Kommentar zum Grundgesetz für die Bundesrepublik Deutschland (AK-GG), Art. 41 (2002), Rn. 15.

37 Vgl. BVerfG, in: NJW 2009, S. 2267 (S. 2268).

38 Josef Isensee, Nemo iudex in causa sua - auch nicht das Parlament?, in: Dieter Dörr / Udo Fink / Christian Hillgruber / Bernhard Kempen / Dietrich Murswiek (Hrsg.), Die Macht des Geistes, Heidelberg 2001, S. 181 - 210, S. 205 f.; Hans-Detlef Horn, Muß die Wahlprüfung Sache des Bundestages sein?, in: Otto Depenheuer / Markus Heintzen / Matthias Jestaedt / Peter Axer (Hrsg.), Staat im Wort, Heidelberg 2007, S. 423 - 439, S. 424 ff.

39 Für eine Verfassungsänderung neuerdings wieder Hans-Detlef Horn, a.a.O. (Fn. 38), S. 435 f.

$40 \mathrm{Vgl}$. zu den Zahlen ebenda, S. 424.

41 Zur Kritik vgl. Hans-Peter Schneider, a.a.O. (Fn. 36), Art. 41 (2002), Rn. 16.

42 Siehe Abschnitt IV Art. VII \$ 112 RVerf. Paulskirche; Art. 27 RV 1871; Art. 31 WRV; vgl. Ernst Rudolf Huber, Deutsche Verfassungsgeschichte seit 1789, Bd. III, Stuttgart 1963, S. 888; Bd. V, Stuttgart 1981, S. 558 f.

43 Vgl. Art. 31 Verf. Baden-Württemberg; Art. 33 BayVerf.; Art. 63 Bbg. Verf.; Art. 9 Hamb. Verf.; Art. 78 Hess. Verf.; Art. 21 I 1 Verf. Meck.-Vorp.; Art. 33 Verf. NRW; Art. 82 Verf. Rh.-Pf.; Art. 75 Verf. Saarland; Art. 45 Sächs. Verf.; Art. 44 I Verf. Sachsen-Anh.; Art. 49 III Thür. Verf.; siehe die Hinweise bei Hermann Rechenberg, a.a.O. (Fn. 24), Art. 41, Rn. 55 ff.

44 Eingehende Darstellung bei Julius Hatschek, Parlamentsrecht des Deutschen Reiches, Berlin 1915, S. 395 ff.; siehe auch Hans Hugo Klein, a.a.O. (Fn. 20), Art. 41, Rn. 18; Gerald Kretschmer, a.a.O. (Fn. 35), $\$ 13$, Rn. 13 ff.

45 Vgl. Hans-Detlef Horn, a.a.O. (Fn. 38), S. 428 f. 
Reichstag und schuf mit dem Wahlprüfungsausschuss nach Art. 31 WRV ein Mischorgan aus Abgeordneten und Berufsrichtern, um der parteipolitischen Instrumentalisierung der Wahlprüfung entgegenzuwirken. ${ }^{46}$ Das Grundgesetz hat diese Tendenz sogar noch verstärkt, indem es das nun allerdings wieder dem Parlament allein vorbehaltene Wahlprüfungsverfahren der Kontrolle durch die Verfassungsgerichtsbarkeit unterstellt hat.

Gewiss sind der weitreichende Ausschluss von Rechtsbehelfen im Vorfeld der Wahl und der rein objektive Charakter der auf die Mandatsrichtigkeit beschränkten Wahlprüfung auch heute noch richtig und sinnvoll. ${ }^{47}$ Es wäre ein misslicher Zustand, würde der Wahlkampf durch eine Vielzahl gerichtlicher Auseinandersetzungen mit Ungewissheiten über die antretenden Parteien belastet und würde jeder Bundestag sozusagen nur unter dem Vorbehalt bundesverfassungsgerichtlicher Bestätigung seine Arbeit aufnehmen können.

Umso mehr ist allerdings kritisch zu fragen, welche politischen Kräfte sich der damit aufgerissenen Rechtsschutzlücke im Verfassungsleben bemächtigt haben. Das führt zurück zum Ausgangspunkt: Angesichts eines gegenüber den Ursprüngen des parlamentarischen Wahlprüfungsrechts völlig veränderten tatsächlichen Kräfteverhältnisses muss in der Parteiendemokratie der Gegenwart alles darauf ankommen, die einfachrechtliche Ausgestaltung des Wahlvorbereitungsverfahrens den verfassungsrechtlichen Gegebenheiten des Rechtsschutzes anzupassen und den parteipolitischen Missbrauch auszuschließen, dem die $\$ \$ 18$ IV und 27 BWahlG in ihrer gegenwärtigen Gestalt Vorschub leisten.

\section{Ausblick: Anstehende Entscheidung des Bundesverfassungsgerichts}

Verschiedene Parteien, die der Bundeswahlausschuss im Juli 2009 nicht als solche anerkannt hat, haben ein parlamentarisches und verfassungsgerichtliches Wahlprüfungsverfahren nach der Bundestagswahl im Herbst angekündigt. Das BVerfG wird dadurch voraussichtlich Gelegenheit erhalten, sich zu der in seiner Rechtsprechung bislang nicht problematisierten Stellung des Bundeswahlausschusses zu äußern. Zwar hat das Gericht nach richtiger, wenn auch nicht unbestrittener Ansicht im Verfahren nach $\$ 48$ BVerfGG keine formelle Normverwerfungskompetenz ${ }^{48}$, aber es kann selbstverständlich auch in diesem Verfahren die einschlägigen Wahlrechtsnormen auf ihre Verfassungsmäßigkeit prüfen ${ }^{49}$ und gegebenenfalls als nichtig behandeln. ${ }^{50}$ Zumindest auf diese Weise kann das Bundesverfassungsgericht den in diesem Beitrag festgestellten Verfassungsverstoß für die Zukunft beseitigen.

46 Vgl. Gerhard Anschütz, Die Verfassung des Deutschen Reichs vom 11. August 1919, Berlin 1933, Art. 31, Nr. 1.

47 Vgl. ausführlich dazu Gerald Kretschmer, a.a.O. (Fn. 35), \$13, Rn. 58 ff.

48 Vgl. Bruno Schmidt-Bleibtreu, a.a.O. (Fn. 31), \$ 48 (2006), Rn. 40; anderer Auffassung Ernst Benda / Eckart Klein, Verfassungsprozessrecht, Heidelberg 2001, Rn. 1176.

49 So geschehen in BVerfGE 16, S. 130 (S. 135 f.); BVerfGE 21, S. 200 (S. 204); BVerfGE 34, S. 81 (S. 95).

50 Vgl. Michael Sachs, Die Bindung des Bundesverfassungsgerichts an seine Entscheidungen, München 1977, S. 403. Da der Wahlprüfungsausschuss des Bundestages kein nach Art. 100 I 1 GG vorlageberechtigtes Gericht ist, könnte die (partielle) Nichtigerklärung der $\$ \$ 18$ IV, 27 BWahlG nur im Rahmen einer abstrakten Normenkontrolle erfolgen. 